\title{
Direct binding of Copine3 with Jab1 activates downstream ErbB2 signaling and motility in SKBr3 breast cancer cells
}

\author{
HYE YOUNG CHOI ${ }^{1}$, NAMMI PARK ${ }^{2}$, JAE BOEM NA $^{1}$, EUN SOOK KO ${ }^{3}$, \\ JAE-YONG PARK $^{4}$ and JAE CHEAL YOO ${ }^{4}$
}

\author{
${ }^{1}$ Department of Radiology, Gyeongsang National University Hospital, Jinju 660-702; ${ }^{2}$ Lab of Aquatic Animal Diseases, \\ College of Veterinary Medicine, Gyeongsang National University, Jinju 660-701; ${ }^{3}$ Department of Radiology, \\ Samsung Medical Center, Sungkyunkwan University School of Medicine, Seoul 135-710; ${ }^{4}$ School of Biosystem \\ and Biomedical Science, College of Health Science, Korea University, Seoul 136-703, Republic of Korea
}

Received September 17, 2015; Accepted November 2, 2015

DOI: $10.3892 /$ or.2015.4472

\begin{abstract}
Copine3, a known calcium-dependent membrane binding protein, contains two tandem $\mathrm{C} 2$ domains and an A domain. This protein has been shown to interact with receptor tyrosine kinase 2 (ErbB2), but little is known concerning the physiological function of Copine3. To better understand its cellular function, we carried out a yeast twohybrid screen to find Copine3 binding partners. Among the identified proteins, Jun activation domain-binding protein 1 (Jab1) appears to directly interact with Copine3. This physical interaction between Copine 3 and Jab1 as well as the specific binding regions of both proteins were confirmed in vitro and in vivo. Our results also demonstrate that binding of Copine 3 to ErbB2 is increased when Jab1 is overexpressed in SKBr3 breast cancer cells. Furthermore, two ErbB2 downstream signaling proteins [phosphatidylinositol 3 (PI3) kinase and protein kinase B (AKT)] were also activated by Jab1 overexpression in these cells. These data suggest that binding of Copine 3 and Jab1 regulates, at least to some extent, the ErbB2 signaling pathway. Moreover, overexpression of both Copine 3 and Jab1 in SKBr3 cells effectively increased cellular migration. Collectively, our findings indicating that Jab1 enhances the ErbB2 binding ability of Copine3, further activating the ErbB2 signaling pathways involved in breast cancer cell pathogenesis.
\end{abstract}

\section{Introduction}

Copines are a family of C2 domain-containing calcium-dependent lipid-binding proteins first identified in Paramecium tetraurelia and are evolutionally conserved from Arabidopsis to Homo sapiens (1). To date, nine human Copine family

Correspondence to: Dr Jae Cheal Yoo, School of Biosystem and Biomedical Science, College of Health Science, Korea University, Seoul 136-703, Republic of Korea

E-mail: jcyoo70@korea.ac.kr

Key words: Copine3, Jab1, ErbB2, SKBr3 cells, wound healing assay members have been identified $(2,3)$. The various functions of this protein family have been shown to be related to cell growth, cancer development and neuronal differentiation (4-6). Copine3, a member of this family, is known to be a membrane binding protein with two tandem $\mathrm{C} 2$ domains, designated $\mathrm{C} 2 \mathrm{~A}$ and $\mathrm{C} 2 \mathrm{~B}$, at the $\mathrm{N}$-terminus followed by an 'A domain' in the C-terminal region (7). Previously, Copine3 was shown to bind to the phosphorylated Tyr1248 of receptor tyrosine kinase 2 (ErbB2), indicating that it may play a regulatory role in ErbB2dependent breast cancer cell motility (8). However, the full mechanism of Copine3 in ErbB2 function is largely unknown.

Jun activation domain-binding protein 1 (Jab1) was originally cloned as a co-activator of activator protein 1 (AP-1) (9). Jab1, also named Cops5, is the fifth component of the COP9 signalosome complex (10), and is evolutionarily conserved in mammals, plants and yeast (11). Furthermore, the deletion of Jab1 in mice results in early embryonic lethality due to impaired cellular proliferation and accelerated apoptosis $(12,13)$. It has also been shown that Jab1 is involved in cell cycle progression, apoptosis, signal transduction and proliferation and is overexpressed in various types of cancer, including breast cancer (14-16).

In addition, several binding partners of Jab1 have been reported, further widening its function. For example, following interaction with Jab1, cyclin-dependent kinase inhibitor 1B (p27 ${ }^{K i p l}$ ) and tumor-suppressor p53 are degraded in the proteasome $(17,18)$. In contrast, Jab1 appears to stabilize hypoxia-inducible factor $1 \alpha$ (HIF-1 $\alpha)$ activation as well as the c-Jun/AP-1 complex $(9,19)$. The binding of Jab1 to its various partners is likely mediated by one of its four binding domains, which include a Jun binding domain at the N-terminus followed by an Mprlp and PAD1p N-terminal (MPN) domain. Other proteins with this MPN domain include proteasome regulatory subunits, eukaryotic initiation factor 3 (eIF3) subunits, and regulators of transcription factor expression and function (20). A nuclear export signal and p27 binding domain are also found in the C-terminal region of Jab1 (14). The presence of these various binding motifs likely mediates the binding of Jab1 to a number of its protein and protein complex partners (21).

In the present study, we have shown that Copine 3 binds to Jab1 in vitro and in vivo, and have further investigated the 
binding region between these two proteins. Furthermore, we also provide data supporting the ability of Jab1 to regulate Copine3-mediated ErbB2 signaling activity, whereby Jab1/ Copine 3 binding appears to increase ErbB2 protein activity as well as downstream cellular migration in SKBr3 breast cancer cells. To our knowledge, this is the first time Copine3/Jab1 binding and its downstream effects have been investigated.

\section{Materials and methods}

Plasmid constructs and mutagenesis. Full-length Copine3 cDNA (GenBank Accession No. NM_003909) was obtained using RT-PCR with RNA isolated from HEK 293T cells. The full-length clone was generated using this cDNA as a template via a PCR-based gateway cloning method previously described (22). C2A, C2B and A domain mutants were obtained using directed PCR in conjunction with primers specific for each region, thus deleting the other domains in the full-length cDNA transcript. Full-length Jab1 cDNA (GenBank Accession No. NM_006837) was obtained from the Korea Research Institute of Bioscience and Biotechnology (KRIBB; Daejoen, Korea; hMU009054). N-, MPN and C-terminal domains mutants were obtained using PCR primers specific for each domain. The resulting PCR products were cloned into the destination vector pDEST-AD-GFP using the gateway cloning system (Invitrogen).

Cell culture. The HEK 293T, COS7, SKBr3 and human breast cancer cell lines used in the present study were all maintained in Dulbecco's modified Eagle's medium (DMEM) supplemented with $1 \%$ penicillin-streptomycin and $10 \%$ fetal bovine serum $(\mathrm{FBS})$ at $37^{\circ} \mathrm{C}$ with $5 \% \mathrm{CO}_{2}$.

Yeast two-hybrid assay. The Copine3 gene was ligated into pGBKT7, which encodes a GAL4 DNA binding domain (BD), while the Jab1 gene was cloned into pGADT7, which encodes an activation domain (AD). To assess the protein-protein interaction between Copine 3 and Jab1, both BD/Copine3 and $\mathrm{AD} / \mathrm{Jab} 1$ were co-transformed into the yeast strain AH109. The transformed yeast was then cultured in SD synthetic medium lacking leucine, tryptophan and histidine.

Adenovirus amplification and infection. Adenovirus was prepared and propagated in HEK 293A cells using the ViraPower $^{\mathrm{TM}}$ Adenoviral Expression System (Invitrogen). pDEST-AD-GFP- and pDEST-AD-mcherry-tagged genes expression vectors were transfected into HEK 293A cells to obtain adenovirus particles. After 7-10 days, virus particles were harvested from the cells and media, followed by purification via centrifugation at 3,000 rpm for $15 \mathrm{~min}$. For adenoviral infection, COS7 or SKBr3 cells were plated into 6-well plates at a density of $1 \times 10^{5}$ cells $/ \mathrm{ml}$ and infected with adenovirus at a multiplicity of infection (MOI) of 100. The infected cells were then incubated for $48 \mathrm{~h}$ at $37^{\circ} \mathrm{C}$.

Confocal microscopy. For our imaging analysis, we used the COS7 cells infected with GFP-Copine 3 and mcherry-Jab1 genes. Further, several randomly chosen fields from multiple wells of cells were photographed using a confocal microscope (Olympus FluoView FV1000).
Western blot analysis and immunoprecipitation (IP). HEK 293T and SKBr3 cells were lysed with RIPA buffer $(50 \mathrm{mM}$ Tris- $\mathrm{HCl}, \mathrm{pH} 7.4,150 \mathrm{mM}$ EDTA, $1 \mathrm{mM}$ PMSF and 1\% NP-40) containing a protease-inhibitor cocktail. Whole-cell lysates were incubated on ice for $30 \mathrm{~min}$ and then cleared at $20,000 \mathrm{xg}$ for $20 \mathrm{~min}$ at $4^{\circ} \mathrm{C}$. For the IP analysis, the resulting supernatants were incubated with the indicated antibodies for $3 \mathrm{~h}$, at $4^{\circ} \mathrm{C}$ with an additional incubation for $2 \mathrm{~h}$ after the addition of protein $\mathrm{A} / \mathrm{G}$ plus agarose (Santa Cruz Biotechnology). The immunocomplexes captured in the agarose gel were then washed three times with RIPA buffer and eluted by boiling with SDS gel-loading buffer. The immunoprecipitates were then analyzed by western blotting.

For the western blot analysis, the supernatants were separated by SDS-PAGE using $10 \%$ gels and blotted onto PVDF membranes. The blots were then probed with antiJab1 (1:1,000), anti-Copine3 (1:1,000) (both from Santa Cruz Biotechnology), anti-ErbB2 (1:2,000, Abcam), anti-PI3 kinase (1:1,000), anti-phospho-PI3 kinase $(1: 1,000)$ (both from Cell Signaling), anti-AKT1/2/3 (1:1,000), anti-phospho-AKT1/2/3 (S473; 1:1,000) and anti-GAPDH (1:3,000) (all from Santa Cruz Biotechnology) antibodies. Blots were then washed and incubated with horseradish peroxidase (HRP)-conjugated anti-mouse or anti-rabbit secondary antibodies, followed by additional washing and the detection with enhanced chemiluminescence (ECL; AbFrontier).

Wound-healing assay. SKBr3 cells (untreated, GFPCopine3- and mcherry-Jab-infected) were inoculated into 6 -well plates, and a $100 \mu 1$ pipette tip was used to create a wound line across the cell monolayer. The cells that moved into the interspace of the scratched wound line were counted $48 \mathrm{~h}$ later using a phase contrast microscope. This assay was performed in triplicate.

Statistical analysis. Statistical analyses were performed with Origin8.0 software using an analysis of variance (ANOVA). Statistical significance was set at $\mathrm{P}<0.005$. Data are reported as the means \pm standard deviation (SD) of three independent experiments.

\section{Results}

Protein-protein interaction between Copine 3 and Jabl in vitro and in vivo. In order to identify novel Copine 3 binding proteins, we performed a yeast two-hybrid screening using Copine 3 as bait. One of the positive clones was determined to be Jab1 (data not shown). This protein-protein interaction between Copine 3 and Jabl was also confirmed using Jab1 as bait in a yeast two-hybrid assay (Fig. 1A) as well as an in vitro binding assay performed in $293 \mathrm{~T}$ cells (Fig. 1B). These data clearly show that Copine3 binds directly to Jab1. Next, the interaction between these two proteins was further confirmed with confocal microscopy of Ad-mcherry-Jab1 and Ad-GFP-Copine3 infected COS7 cells. It appears that Jab1 localizes to the nucleus and cytosol region, particularly around the membrane, while Copine 3 localizes to the around nucleus and cytosol (Fig. 1C). The areas where these two proteins are co-localized in the membrane are marked with an arrow (Fig. 1C). 
A

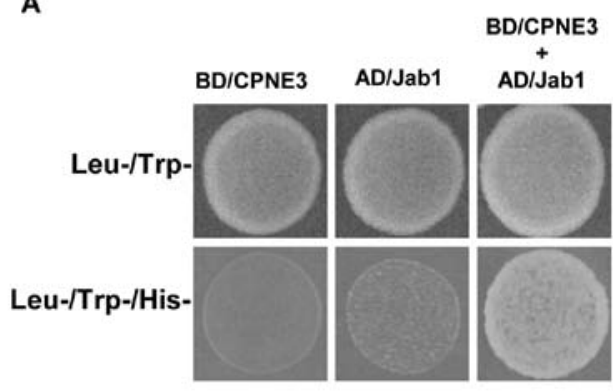

B

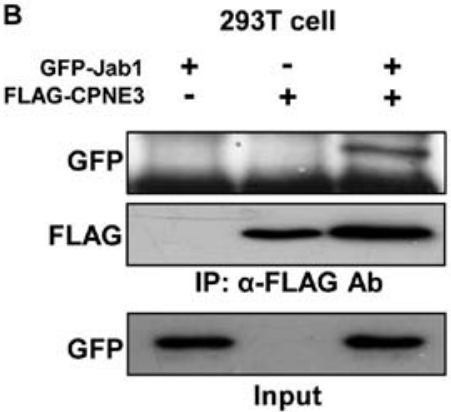

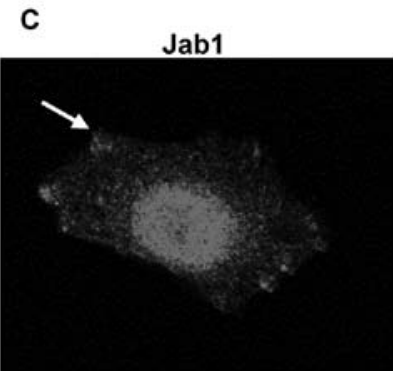

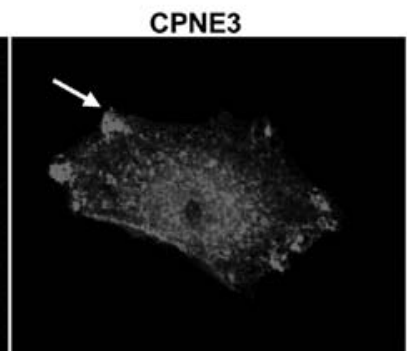

cos7 cell

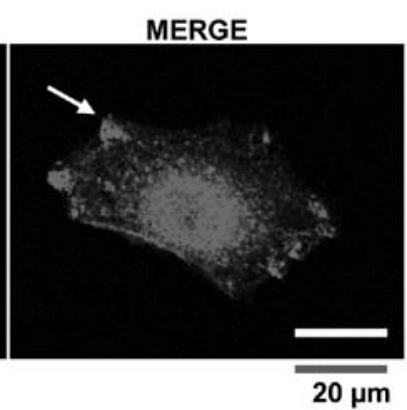

D

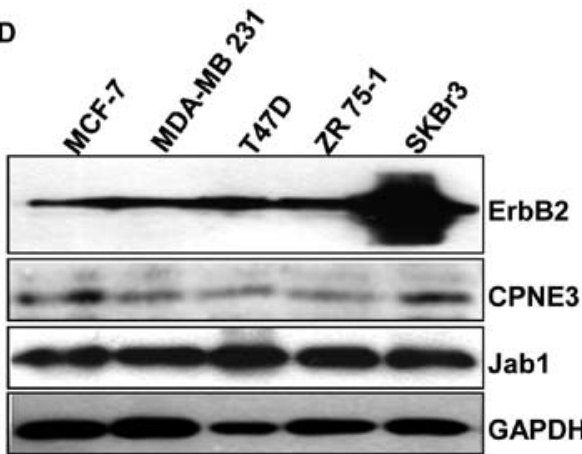

\section{SKBr3 cell}

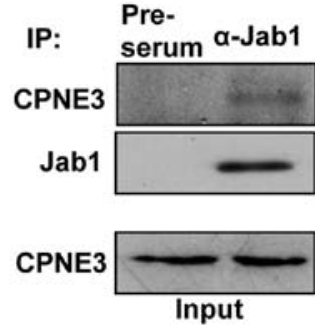

Figure 1. Interaction of Jab1 with Copine3. (A) Yeast two-hybrid assay of the interaction between Jab1 and Copine3. (B) Co-immunoprecipitation of Jab1 with Copine3. Flag-Copine3 and GFP-Jab1 were co-transfected into HEK 293T cells. (C) Co-localization of mcherry-Jab1 and GFP-Copine3 in COS7 cells. The white arrow indicates co-localization around the plasma membrane of the cells (scale bar, $20 \mu \mathrm{m}$ ). (D) ErbB2, Jab1 and Copine3 protein expression in several breast cancer cell types. Expression was analyzed by western blotting with GAPDH being used as a loading control. (E) Endogenous Jab1 associates with Copine 3 in SKBr3 cells.

We then evaluated the endogenous protein expression of ErbB2, Copine3 and Jab1 in several breast cancer cell types (Fig. 1D). To investigate the interaction between Jab1 and Copine 3 and the effects of this interaction on ErbB2/Copine3-related pathways in breast cancer, endogenous co-IP experiments were carried out in SKBr3 cells. Notably, IP with anti-Jab1 and pre-serum antibodies, followed by western blotting with an anti-Copine3 antibody, showed that Jab1 is in fact associated with Copine3 in this cell type (Fig. 1E).

Identification of the binding regions involved in the Jabl/ Copine 3 interaction. To identify the specific binding regions involved for both proteins involved in the Jab1/Copine3 interaction, the N-terminus, MPN domain and C-terminus of Jab1 as well as the $\mathrm{C} 2 \mathrm{~A}, \mathrm{C} 2 \mathrm{~B}$, and $\mathrm{A}$ domains of Copine 3 were isolated and N-terminally tagged with GFP (Fig. 2A and B for Jab1 and Copine3, respectively). These truncated mutants were then co-transfected into $293 \mathrm{~T}$ cells with either FLAG-Copine3 (for the Jab1 mutants) or FLAG-Jab1 (for the Copine3 mutants). Our data indicate that, while Copine 3 does not bind with the
MPN domain of Jab1, it does interact with both the $\mathrm{N}$ - and C-terminal domains (Fig. 2A), indicating that the tertiary structure of Jab1 may be important during this binding interaction. Notably, Jab1 appears to bind only with the C2A domain of Copine3 (Fig. 2B), demonstrating that this region contains the binding site of Jab1. Generally, the $\mathrm{C} 2$ domains in Copine 3 are known for their $\mathrm{Ca}^{++}$and lipid binding capabilities; however, our data indicate that at least the $\mathrm{C} 2 \mathrm{~A}$ domain also functions as a putative binding domain.

Jab1/Copine3 binding induces interaction between Copine3 and ErbB2, activating downstream ErbB2 signaling pathways in SKBr3 cells. It has been reported that Copine 3 interacts with ErbB2 in various breast cancer cell types (8). To investigate the effect of Jab1 binding on the interaction between Copine3 and ErbB2, co-IP experiments were carried out in SKBr3 cells overexpressing Jab1 (Fig. 3A). It appears that the interaction between Copine 3 and ErbB2 was markedly increased when Jab1 was overexpression in this cell type. In addition, to further confirm ErbB2 pathway activation, various downstream 

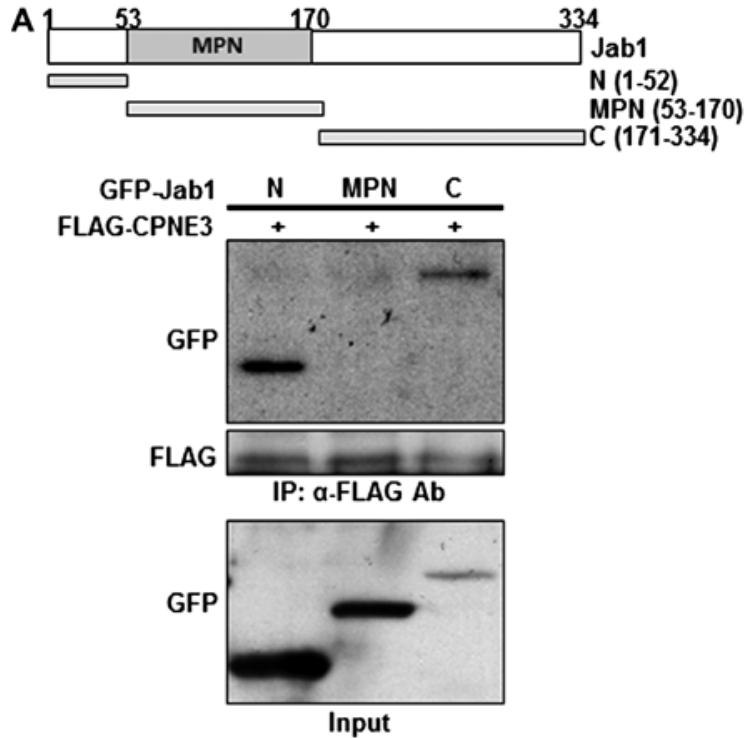

B

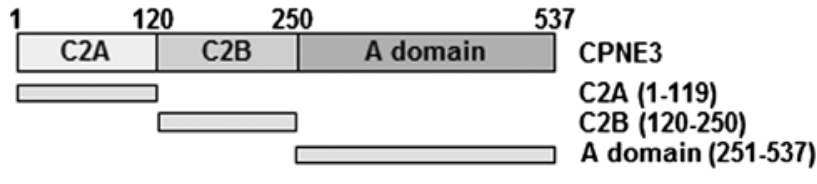

Figure 2. Binding regions of Jab1 and Copine3 involved in their interaction. (A) Schematic of the truncated Jab1 mutants used in this experiment. GFP-Jab1 truncated mutant constructs were transfected with FLAG-Copine3 construct into 293T cells and then co-immunoprecipitated with an anti-FLAG antibody. (B) Schematic of the truncated Copine3 mutants used in this experiment. GFP-Copine3 truncated mutant constructs were transfected with FLAG-Jab1 construct into 293T cells and then co-immunoprecipitated with anti-GFP antibody. Input shows 5\% of the total lysate used for the immunoprecipitation.

A
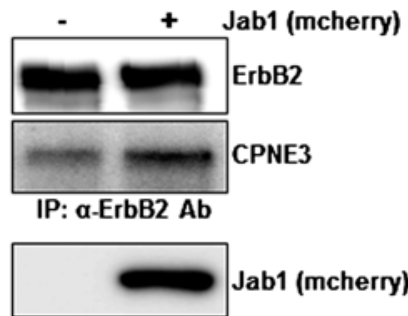

B

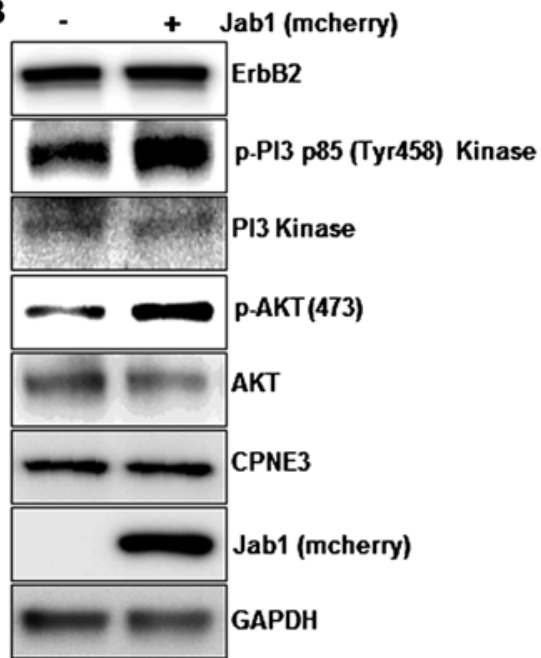

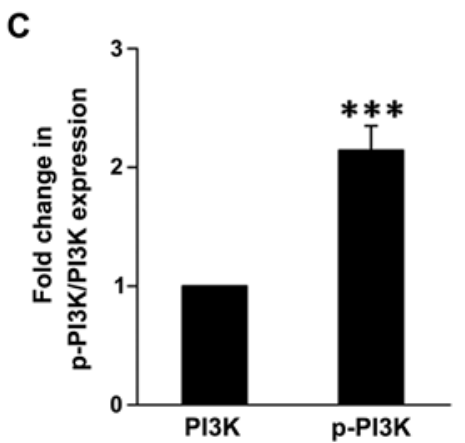

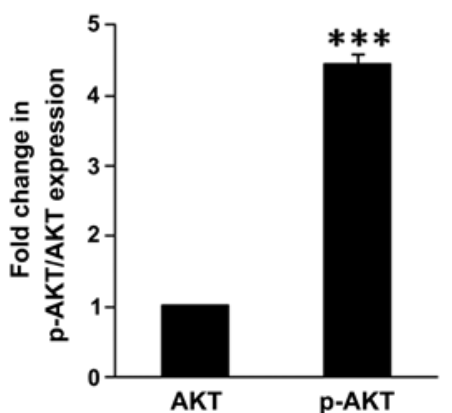

Figure 3. Jab1/Copine3 binding increases the interaction between ErbB2 and Copine3, activating ErbB2-related downstream signaling proteins in SKBr3 cells. (A) Co-immunoprecipitation of ErbB2 with Copine3 with/without Jab1. Ad-mcherry construct (control) only or Ad-mcherry-tagged Jab1 were infected into SKBr3 cells. (B) Under these conditions, the expression and phosphorylation status of two ErbB2 downstream proteins, PI3 p85 kinase and AKT, were analyzed by western blotting. GAPDH was used as the loading control. (C) The Bar graphs show the mean percentages of phosphor-PI3 p85 (Tyr458) and phosphor-Akt (S473) of (B) from three independent experiments $\left({ }^{* * *} \mathrm{P}<0.001\right.$; ANOVA).

signaling proteins were investigated. Notably, the levels of phosphorylated PI3 p85 kinase and AKT appear to increase when Jab1 is overexpressed in SKBr3 cells (Fig. 3B and C), indicating that high levels of Jab1, and thus an increase in 
A
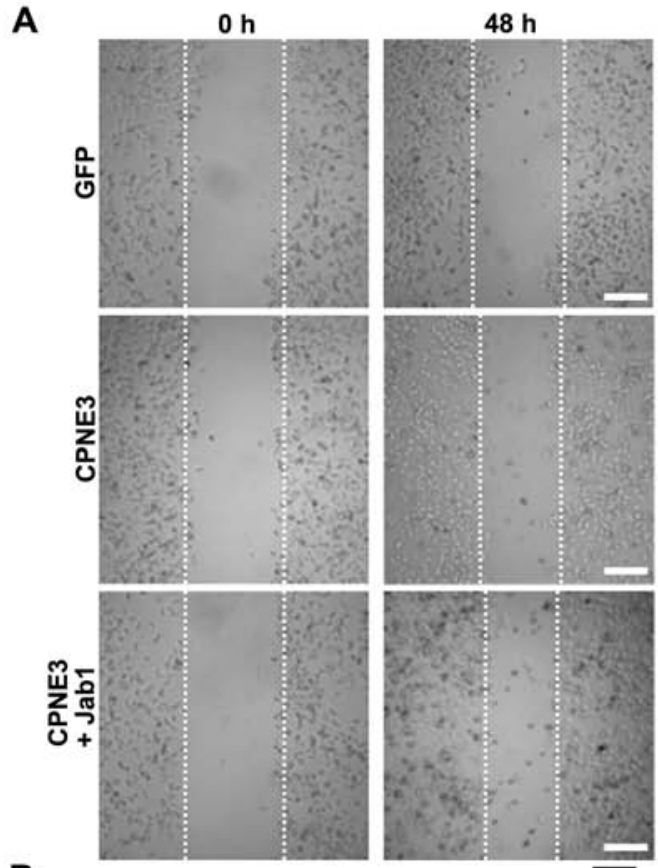

B
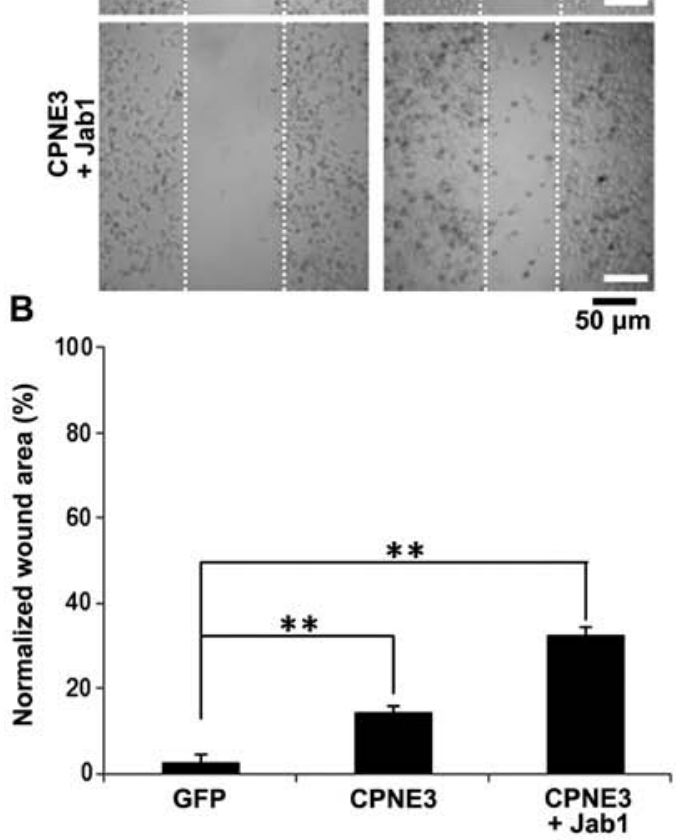

C

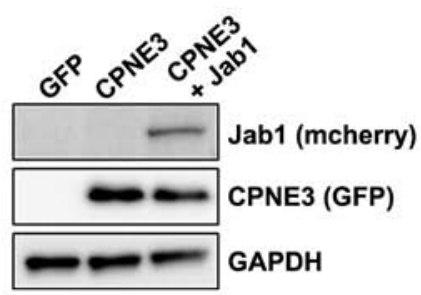

Figure 4. Overexpression of Jab1 with Copine3 effectively induces a higher level of SKBr3 cellular migration. (A) Representative image of the migration induced in SKBr3 cells infected with Ad-GFP (control), Ad-GFP-Copine3 and Ad-GFP-Copine3 + Ad-mcherry-Jab1 after $48 \mathrm{~h}$ of the cell monolayer was wounded (scale bar, $50 \mu \mathrm{m}$ ). (B) y-axis of histogram shows recover area after 'wound' in each group after $48 \mathrm{~h}$. The percentages of normalized cell count are shown in the bar graph as mean \pm SD from three independent experiments. An ANOVA was used for statistical analysis. ${ }^{* *} \mathrm{P}<0.005$ for Copine 3 vs. the GFP control and Copine3 + Jab1 vs. the GFP control (C) Expression levels of Jab1 and Copine 3 were analyzed by western blotting. GAPDH was used as the loading control.

Jab1/Copine3 binding, activate ErbB2-related signaling in this cell type. Based on these data, we conclude that Jab1 regulates ErbB2 downstream signaling pathway via binding to Copine3 and inducing the interaction between Copine3 and ErbB2.

Jab1/Copine3 binding activates tumor cell migration in SKBr3 cells. In addition to investigating the activation of downstream proteins involved in the ErbB2-mediated signaling cascade, we also examined the effect of Jab1/Copine3 binding on ErbB2-dependent breast cancer cell motility. To do so, we utilized a wound-healing assay using SKBr3 cells. One day following infection with Ad-GFP, Ad-Copine3 or Ad-Copine3 + Ad-Jab1, we created a 'wound' in the monolayered SKBr3 cells. Cell migration into the wound was evaluated after $48 \mathrm{~h}$ (Fig. 4A). Basal migration of the GFP infected control cells was low. Not surprisingly, the number of migrating cells into the wound area increased $\sim 15 \%$ in the cells overexpressing Copine3 alone (Fig. 4A and B). However, the number of cells that migrated into the wound area was $\sim 35 \%$ higher in the Copine 3 and Jab1 co-infected cells compared to the controls. The expression levels of Copine 3 and Jab1 were also confirmed using western blot analysis (Fig. 4C). Notably, in this analysis, GAPDH protein was similarly expressed in each group. Taken together, our data show that overexpression of Jab1 in conjunction with Copine 3 overexpression, and their subsequent binding, markedly increases SKBr3 cell migration.

\section{Discussion}

In the present study, we revealed a novel binding relationship between Copine 3 and Jab1. This interaction was first demonstrated with a yeast two-hybrid screen using Copine3 as bait. Notably, we then confirmed this interaction in a reverse yeast two-hybrid screen with Jab1 as bait as well as using in vitro and in vivo assays. These assays further confirmed the co-localization of Copine 3 and Jab1 and direct binding between the two proteins in breast cancer cells. The decision to focus on the role of these proteins specifically in breast cancer cells stemmed from a previous study that Copine 3 expression correlates with ErbB2 amplification in breast cancer and preferentially binds to the pTyr1248 residue of this oncogene (8). This previous study also indicated that this Copine3/ErbB2 interaction occurs at the plasma membrane. Furthermore, Jab1 protein is also largely expressed in invasive breast cancer (23). Thus, the proximity of these proteins in breast cancer cells, confirmed in the present study, spurred us to investigate their possible influence on each other's expression and function. Notably, in a preliminary analysis, we did not find any evidence supporting the direct binding of Jab1 with ErbB2 (data not shown), further focusing our investigation on the relationship between Jab1 and Copine3.

It is generally known that the A domain found in members of the Copine family is the primary protein binding domain involved in regulation, while the $\mathrm{C} 2$ domains are typically related to $\mathrm{Ca}^{++}$and membrane binding (2). However, Benes et al revealed that the $\mathrm{C} 2$ domain of protein kinase $\mathrm{C} \delta$ (PKCס) directly binds to phosphotyrosine peptides in a sequencespecific manner (24). In the present study, we have shown that the $\mathrm{C} 2 \mathrm{~A}$ domain of Copine 3 is the only region involved in Jab1 binding. As this is one of the few studies identifying non- $\mathrm{Ca}^{++} /$membrane-dependent $\mathrm{C} 2$ domain-mediated binding, additional study is necessary to determine the full function of this binding motif.

Furthermore, Jab1 has been shown to bind to and degrade p2 $7^{\text {Kipl }}$ and p53, while in other contexts it can stabilize HIF- $1 \alpha$ activation and the c-Jun/AP-1 complex (9,17-19). The binding domains involved in these Jabl functions are 
found throughout the structure and include a Jun binding domain at the N-terminus, an MPN domain, as well as a p27 binding domain at the C-terminus (17-19). Generally, the function of the MPN domain has been related to ubiquitin isopeptidase/deubiquitinase in the ubiquitin-based signaling and protein turnover pathways in eukaryotes (20). Our data indicate that Copine 3 preferentially binds with the $\mathrm{N}$ - and C-terminal regions of Jab1, thereby excluding the MPN domain. Notably, this distinct exclusion of MPN domain binding possibly indicates Jab1 binding-mediated activation of Copine3. In fact, our results show that binding of Jab1 and Copine3 activates Copine3/ErbB2 binding as well as the downstream ErbB2 signaling cascade and ErbB2-mediated cell migration. We believe that this Jab1/Copine3-mediated activation may involve Jab1 binding-induced conformation changes in the tertiary structure of Copine3, making this protein more accessible and/or more susceptible to ErbB2 binding. Additional studies identifying the specific binding sites and structural organization of the Jab1/Copine3 complex are warranted.

In conclusion, we have investigated the binding complex formed by Jab1 and Copine 3 in breast cancer cells. Our data indicate that this binding complex not only forms in breast cancer cells, but also influences the ErbB2 binding capabilities of Copine3. It is likely that Copine 3 is stabilized through the binding of Jab1, potentially allowing it to bind more strongly to ErbB2. Thus, Copine3 appears to be the link between Jab1 expression and ErbB2-mediated downstream signal pathways and cellular motility. The present study is the first to describe the Copine3/Jab1 interaction and further enhances our understanding of the signaling cascades involved in breast cancer.

\section{Acknowledgements}

The present study was supported by the Basic Science Research Program (NRF-2012R1A1A2005035) and the Bio-Synergy Research Project (NRF-2014M3A9C4066463) through the National Research Foundation of Korea.

\section{References}

1. Creutz CE, Tomsig JL, Snyder SL, Gautier MC, Skouri F, Beisson $\mathrm{J}$ and Cohen J: The copines, a novel class of $\mathrm{C} 2$ domaincontaining, calcium-dependent, phospholipid-binding proteins conserved from Paramecium to humans. J Biol Chem 273: 1393-1402, 1998.

2. Tomsig JL and Creutz CE: Copines: A ubiquitous family of $\mathrm{Ca}^{2+}$ dependent phospholipid-binding proteins. Cell Mol Life Sci 59: 1467-1477, 2002.

3. Maitra R, Grigoryev DN, Bera TK, Pastan IH and Lee B: Cloning, molecular characterization, and expression analysis of Copine 8 . Biochem Biophys Res Commun 303: 842-847, 2003.

4. Yang S, Yang H, Grisafi P, Sanchatjate S, Fink GR, Sun Q and Hua J: The BON/CPN gene family represses cell death and promotes cell growth in Arabidopsis. Plant J 45: 166-179, 2006.
5. Ramsey CS, Yeung F, Stoddard PB, Li D, Creutz CE and Mayo MW: Copine-I represses NF-kappaB transcription by endoproteolysis of p65. Oncogene 27: 3516-3526, 2008.

6. Park N, Yoo JC, Ryu J, Hong SG, Hwang EM and Park JY: Copinel enhances neuronal differentiation of the hippocampal progenitor HiB5 cells. Mol Cells 34: 549-554, 2012.

7. Whittaker CA and Hynes RO: Distribution and evolution of von Willebrand/integrin A domains: Widely dispersed domains with roles in cell adhesion and elsewhere. Mol Biol Cell 13: 3369-3387, 2002.

8. Heinrich C, Keller C, Boulay A, Vecchi M, Bianchi M, Sack R, Lienhard S, Duss S, Hofsteenge J and Hynes NE: Copine-III interacts with ErbB2 and promotes tumor cell migration. Oncogene 29: 1598-1610, 2010.

9. Claret FX, Hibi M, Dhut S, Toda T and Karin M: A new group of conserved coactivators that increase the specificity of AP-1 transcription factors. Nature 383: 453-457, 1996.

10. Chamovitz DA and Segal D: JAB1/CSN5 and the COP9 signalosome. A complex situation. EMBO Rep 2: 96-101, 2001.

11. Wei N, Tsuge T, Serino G, Dohmae N, Takio K, Matsui M and Deng XW: The COP9 complex is conserved between plants and mammals and is related to the $26 \mathrm{~S}$ proteasome regulatory complex. Curr Biol 8: 919-922, 1998.

12. Tomoda K, Yoneda-Kato N, Fukumoto A, Yamanaka S and Kato JY: Multiple functions of Jab1 are required for early embryonic development and growth potential in mice. J Biol Chem 279: 43013-43018, 2004.

13. Tian L, Peng G, Parant JM, Leventaki V, Drakos E, Zhang Q, Parker-Thornburg J, Shackleford TJ, Dai H, Lin SY, et al: Essential roles of Jab1 in cell survival, spontaneous DNA damage and DNA repair. Oncogene 29: 6125-6137, 2010.

14. Shackleford TJ and Claret FX: JAB1/CSN5: A new player in cell cycle control and cancer. Cell Div 5: 26, 2010.

15. Wei N, Serino G and Deng XW: The COP9 signalosome: More than a protease. Trends Biochem Sci 33: 592-600, 2008.

16. Wang J, Barnes RO, West NR, Olson M, Chu JE and Watson PH: Jabl is a target of EGFR signaling in ERalpha-negative breast cancer. Breast Cancer Res 10: R51, 2008

17. Tomoda K, Kubota Y, Arata Y, Mori S, Maeda M, Tanaka T, Yoshida M, Yoneda-Kato N and Kato JY: The cytoplasmic shuttling and subsequent degradation of $\mathrm{p} 27^{\mathrm{Kipl}}$ mediated by Jab1/ CSN5 and the COP9 signalosome complex. J Biol Chem 277: 2302-2310, 2002

18. Lee EW, Oh W and Song J: Jab1 as a mediator of nuclear export and cytoplasmic degradation of p53. Mol Cells 22: 133-140, 2006.

19. Bae MK, Ahn MY, Jeong JW, Bae MH, Lee YM, Bae SK, Park JW, Kim KR and Kim KW: Jab1 interacts directly with HIF-1alpha and regulates its stability. J Biol Chem 277: 9-12, 2002.

20. Birol M and Echalier A: Structure and function of MPN (Mpr1/ Pad1 N-terminal) domain-containing proteins. Curr Protein Pept Sci 15: 504-517, 2014.

21. Burger-Kentischer A, Finkelmeier D, Thiele M, Schmucker J, Geiger G, Tovar GE and Bernhagen J: Binding of JAB1/CSN5 to MIF is mediated by the MPN domain but is independent of the JAMM motif. FEBS Lett 579: 1693-1701, 2005.

22. Park JY, Hwang EM, Park N, Kim E, Kim DG, Kang D, Han J, Choi WS, Ryu PD and Hong SG: Gateway RFP-fusion vectors for high throughput functional analysis of genes. Mol Cells 23: 357-362, 2007.

23. Kouvaraki MA, Rassidakis GZ, Tian L, Kumar R, Kittas C and Claret FX: Jun activation domain-binding protein 1 expression in breast cancer inversely correlates with the cell cycle inhibitor p27 ${ }^{\text {Kipl }}$. Cancer Res 63: 2977-2981, 2003.

24. Benes CH, Wu N, Elia AE, Dharia T, Cantley LC and Soltoff SP: The C2 domain of PKCdelta is a phosphotyrosine binding domain. Cell 121: 271-280, 2005. 